\title{
A brief review of procedures and potential problems associated with the diagnosis of porcine reproductive and respiratory syndrome
}

\author{
William L. MengelinG*, Kelly M. LAGer \\ Virology Swine Research Unit, National Animal Disease Center, USDA, Agricultural Research \\ Service, 2300 Dayton Avenue, P.O. Box 70, Ames, Iowa 50010, USA
}

\begin{abstract}
Experience has shown that, for a number of reasons, a diagnosis of porcine reproductive and respiratory syndrome (PRRS) is sometimes difficult. In this review we discuss: (1) field observations and laboratory tests that are useful in arriving at a definitive diagnosis; (2) the impact of so-called atypical PRRS on diagnostic procedures in North America; (3) the means by which diagnostic problems can often be circumvented by appropriate sample selection; and (4) methods used for
\end{abstract} presumptive identification of PRRS virus strains.

porcine reproductive and respiratory syndrome virus / PRRSV / diagnosis / atypical PRRS

Résumé - Procédures et problèmes potentiels associés au diagnostic du syndrome dysgénésique et respiratoire porcin : une brève revue. L'expérience a montré que, pour un certain nombre de raisons, le diagnostic du syndrome dysgénésique et respiratoire porcin (PRRS) est parfois difficile. Dans cet article de synthèse, nous discutons : (1) les observations sur le terrain et les tests de laboratoire utiles pour parvenir à un diagnostic définitif ; (2) l'impact du PRRS appelé "atypique" sur les procédures diagnostiques en Amérique du Nord ; (3) les moyens par lesquels les problèmes diagnostiques peuvent être évités, grâce à une sélection pertinente des échantillons ; et (4) les méthodes utilisées pour l'identification différentielle des souches de virus du PRRS.

syndrome dysgénésique et respiratoire porcin / PRRSV / diagnostic / PRRS atypique

* Correspondence and reprints

Tel.: (1) 515663 7254; fax: (1) 515663 7458; e-mail: wmengeli@ nadc.ars.usda.gov 
Table of contents

1. Introduction ....................... 62

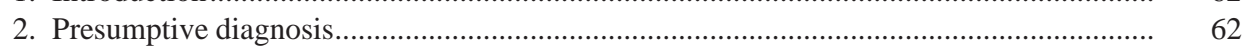

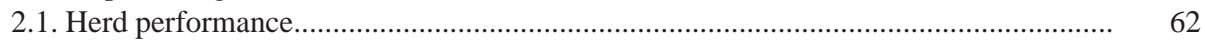

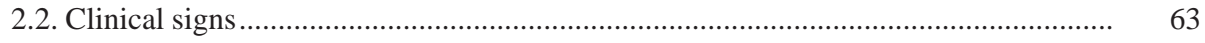

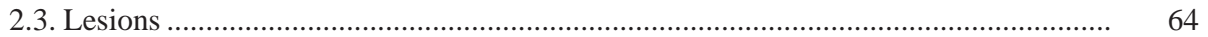

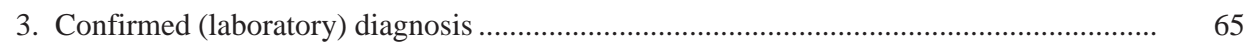

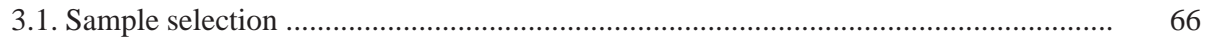

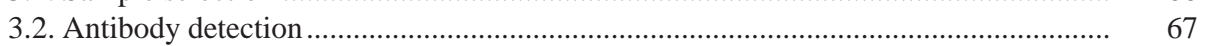

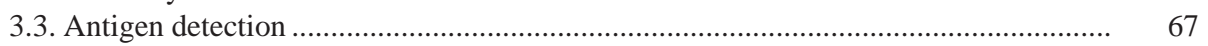

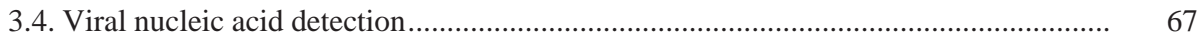

3.5. Infectious virus detection .............................................................................. 68

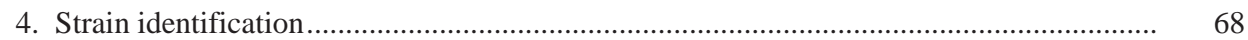

\section{INTRODUCTION}

A timely and unequivocal diagnosis of infectious diseases depends on the ability to recognize the disease in question, regardless of its clinical manifestation at the moment, and to quickly isolate and identify the causative agent. Although these requirements are straightforward in principle, they are, for a variety of reasons, often more difficult to satisfy in practice than in theory. The diagnosis of porcine reproductive and respiratory syndrome (PRRS) can be particularly problematic. The presentation of clinical illness can vary widely depending on the age and relative susceptibility of the infected pig, the involvement of other pathogens, the stage of gestation in the case of reproductive failure, and the relative virulence of the causative strain of PRRS virus (PRRSV). Moreover, some strains of PRRSV are difficult to isolate and identify because they replicate poorly in cell cultures. An additional issue of diagnostic importance is that because attenuated vaccine virus can persist in tissues of vaccinated pigs for weeks and perhaps months it is often necessary to question the significance of virus isolation in vaccinated herds.

In the following review we discuss these and other potential problems encountered in PRRS diagnosis, and the means by which they often can be circumvented or minimized. Most of the information is from observations made with pigs infected with North American strains of PRRSV. We assume that, in general, it also applies to the diagnosis of PRRS caused by European strains of the virus.

\section{PRESUMPTIVE DIAGNOSIS}

A presumptive diagnosis of PRRS can often be made on the basis of herd performance, clinical signs, and lesions.

\subsection{Herd performance}

Several serologic surveys have established that endemic infection with PRRSV is common. However, in the absence of obvious clinical signs there may be little or no evidence to suggest economic impact. A comparison of current and past (that is pre-infection) production records of infected herds may reveal a moderate decrease in growth rate, feed efficiency, or reproductive performance, or in some combination of these or other production parameters; but without a noninfected control group maintained under the same conditions, it may be impossible to determine whether any differences that might be detected are actually 
virus induced. What we do know is that when otherwise healthy pigs are infected with moderately virulent strains of PRRSV under experimental conditions, their performance, such as rate of gain, can be adversely affected, even though visible clinical signs are minimal or absent. It seems reasonable to assume that the same scenario occurs in the field.

\subsection{Clinical signs}

The most commonly described clinical signs associated with PRRS are reproductive failure of gilts and sows, and respiratory tract illness in pigs of all ages, but particularly in young pigs. At one time PRRSV-induced reproductive losses in swine in North America were believed to be limited mainly to late-term abortions, and the delivery, at term or later, of weak and stillborn pigs and fetuses that had died late in gestation. Results of experiments using several strains of PRRSV isolated relatively soon after PRRS was first recognized in North America were generally consistent with field observations and suggested that salient features of the reproductive facet of the syndrome were: 1) pregnant gilts and sows typically had moderate to mild and transient, or no, clinical signs other than reproductive failure; and 2) reproductive failure was limited mainly to the effects of the virus on late-term gestation.

However, the clinical picture changed dramatically in North America in the fall of 1996 when an epidemic of PRRS characterized by gilt and sow mortality and reproductive failure (often abortions) in early as well as late gestation was reported in southeastern Iowa. In some affected herds, the prevalence of gilt and sow death was $5 \%$ or more and the name sow abortion and mortality syndrome (SAMS) was proposed. A questionnaire sent to swine practitioners throughout North America soon after initial recognition of the south-eastern Iowa epidemic revealed that clinically similar epidemics had been recognized elsewhere [7].

At first the cause of these epidemics was in question. Some veterinarians and swine producers feared that we were facing a new "mystery disease" with a different cause, but in other respects analogous to the situation encountered when PRRS first appeared in the United States in the late 1980's. In retrospect it appears that the initial confusion about the cause of the clinically severe epidemics (now generally referred to as "acute" or "atypical" PRRS rather than SAMS) was simply the result of using sampling techniques and testing procedures that, for reasons addressed in a subsequent section, had become less reliable. It is now generally believed that atypical PRRS (a name now often used by many for any clinically severe epidemic of PRRS with or without associated gilt and sow mortality) was, and is, caused by strains of PRRSV that increased in virulence, presumably as a result of mutation in the field.

Under experimental conditions this increase in virulence is clearly evident following infection of pregnant gilts. Although all field strains of PRRSV appear to have the potential to cause reproductive failure, only strains isolated from atypical PRRS epidemics have, in our experience, caused severe clinical signs during the acute stage of infection. These include fever (body temperatures sometimes in excess of $41{ }^{\circ} \mathrm{C}$ beginning within 30 hours of initial exposure), recumbency, and partial to total inappetence. Their duration is typically 7 to 14 days. Only one of about 40 pregnant gilts we have infected experimentally have died as a result; however, as mentioned previously, gilt and sow death has been a major part of some field epidemics of atypical PRRS.

The severity of PRRSV-induced respiratory tract disease in young pigs also suggests the emergence of more virulent strains of PRRSV. Under experimental conditions, some of the more recently isolated strains 
of PRRSV cause marked clinical illness and sometimes death in specific-pathogen-free pigs exposed to the virus at 2 to 3 weeks of age. These observations are in contrast to the milder form of clinical illness usually seen in pigs exposed experimentally to strains of PRRSV isolated before the appearance of atypical PRRS. A more detailed description of clinical signs as well as an extensive list of relevant references can be found elsewhere [1].

\subsection{Lesions}

The most characteristic lesions of PRRS are seen in young pigs affected with the respiratory facet of the syndrome. Following experimental infection of healthy young pigs, macroscopic lesions are presented mainly as tan to grey, enlarged lymph nodes, which are often several times the size of normal nodes for the same size pig (Fig. 1) and focal to diffuse areas of pulmonary con- solidation (Fig. 2). However, in the field these lesions are often complicated or obscured by concurrent infections. The degree of pulmonary consolidation seems to be directly related to the relative virulence of the strain of PRRSV to which pigs are exposed. In the most severe experimental cases, lungs may be almost totally consolidated at about 2 weeks after initial exposure. Without complications, resolution begins shortly thereafter, and by 4 weeks after initial exposure the extent of lung involvement has usually decreased markedly. The degree of lymph node enlargement seems to be less related to strain virulence and there may be little if any difference between the size of nodes of pigs exposed to moderately or highly virulent strains. Even attenuated vaccine strains may cause some (albeit much less) lymph node enlargement. Lymph nodes may remain enlarged for 6 or more weeks (Tab. I). There are typically few or no macroscopic lesions of diagnostic importance associated with

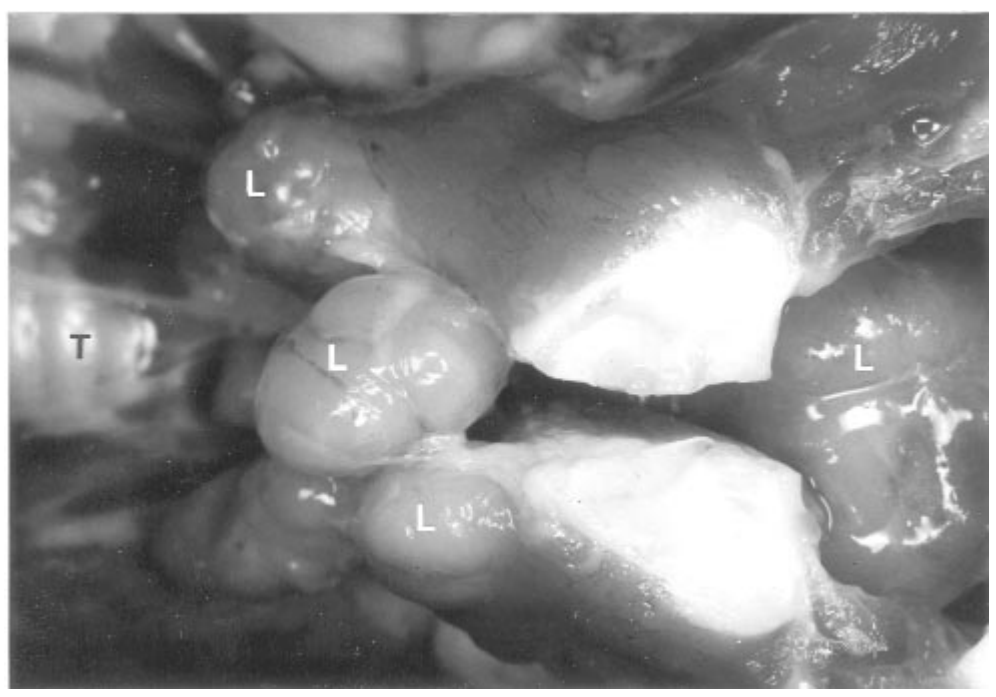

Figure 1. Enlarged lymph nodes (sternal and deep cervical) at the thoracic inlet of a pig exposed oronasally to a virulent strain of porcine reproductive and respiratory syndrome virus 4 weeks before necropsy. The pig was about 7 weeks old at the time of necropsy. The thymus was removed to make the nodes more obvious. $\mathrm{L}=$ lymph nodes; $\mathrm{T}=$ trachea. 


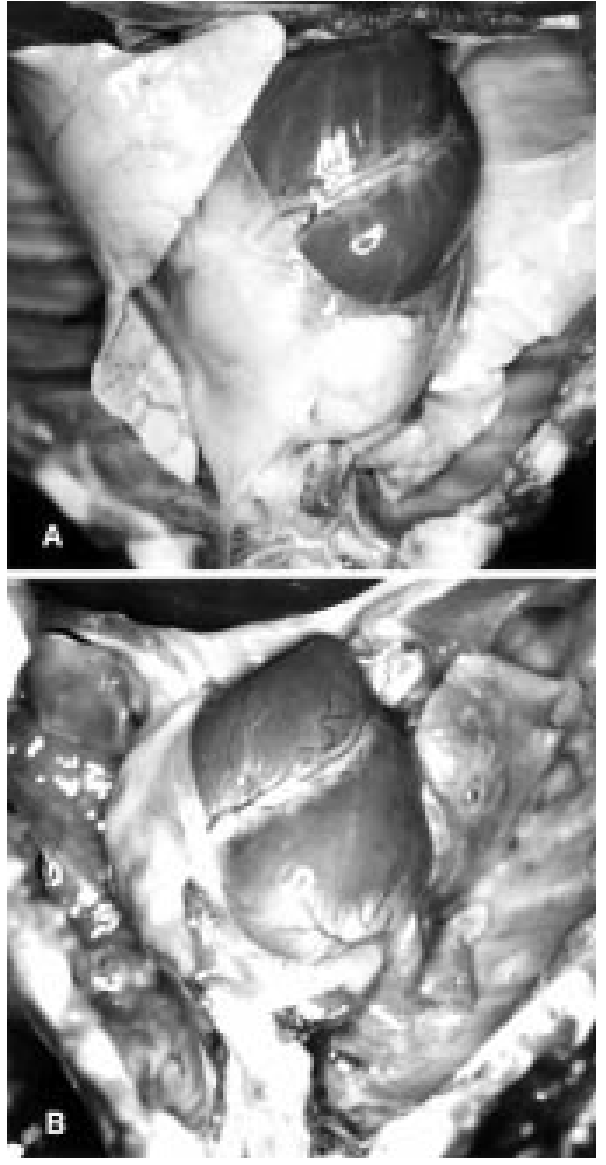

Figure 2. Macroscopic appearance of normal lungs (A) of a pig kept as a noninfected control and of extensively consolidated lungs (B) of a pig exposed to a highly virulent (atypical) strain of a porcine reproductive and respiratory syndrome virus 2 weeks before necropsy. Both pigs were about 5 weeks old at the time of necropsy.

the reproductive facet of PRRS. However, segmental hemorrhages in the umbilical cord of weak liveborn and stillborn pigs are sometimes present.

Microscopic lesions of the most diagnostic value in young pigs are focal to diffuse areas of mixed mononuclear cell infiltration in the lungs (Fig. 3) and follicular
Table I. Effect of porcine reproductive and respiratory syndrome virus (PRRSV) on lymph node weight.

\begin{tabular}{lccc}
\hline $\begin{array}{l}\text { Exposure } \\
\text { Strain }\end{array}$ & $\begin{array}{c}\text { Postexposure } \\
\text { week }\end{array}$ & \multicolumn{2}{c}{ Weight } \\
& & Pigs (kg) & Nodes $(\mathrm{g})$ \\
\hline None & 2 & 7.82 & 1.99 \\
Vaccine & 2 & 6.00 & 2.24 \\
Field & 2 & 8.32 & 6.00 \\
None & 4 & 9.77 & 1.74 \\
Vaccine & 4 & 8.27 & 3.78 \\
Field & 4 & 10.32 & 9.22 \\
None & 6 & 15.82 & 2.79 \\
Vaccine & 6 & 13.05 & 4.44 \\
Field & 6 & 15.55 & 10.01 \\
\hline
\end{tabular}

A total of 18 pigs (3 groups comprising 6 pigs/group) were kept as noninfected controls, or were exposed oronasally to $10^{6}$ median cell culture infective doses of either vaccine (RespPRRS ${ }^{\circledR}$ ) or field (NADC-9, isolated in 1993) strains of PRRSV. Selected lymph nodes (sternal and deep cervical) were weighed to provide a numerical indication of the generalized lymph node enlargement caused by infection with the respective strains of PRRSV. Each weight value represents the average of 2 pigs. None of the pigs had any obvious clinical signs during the study.

hyperplasia of lymph nodes. Affected gilts and sows may have a mild to moderate lymphoplasmacytic endometritis and myometritis. A more detailed description of lesions as well as an extensive list of relevant references can be found elsewhere [1].

\section{CONFIRMED (LABORATORY) DIAGNOSIS}

A confirmed diagnosis of PRRS is generally considered to have been established when PRRSV is identified in samples submitted from presumed cases of the disease. However, for a variety of reasons, isolation of infectious virus can be difficult. If a particular laboratory has experience with PRRSV and has available all of the necessary diagnostic reagents, the key to a successful diagnosis is likely to depend mainly on selection and submission of appropriate samples. 


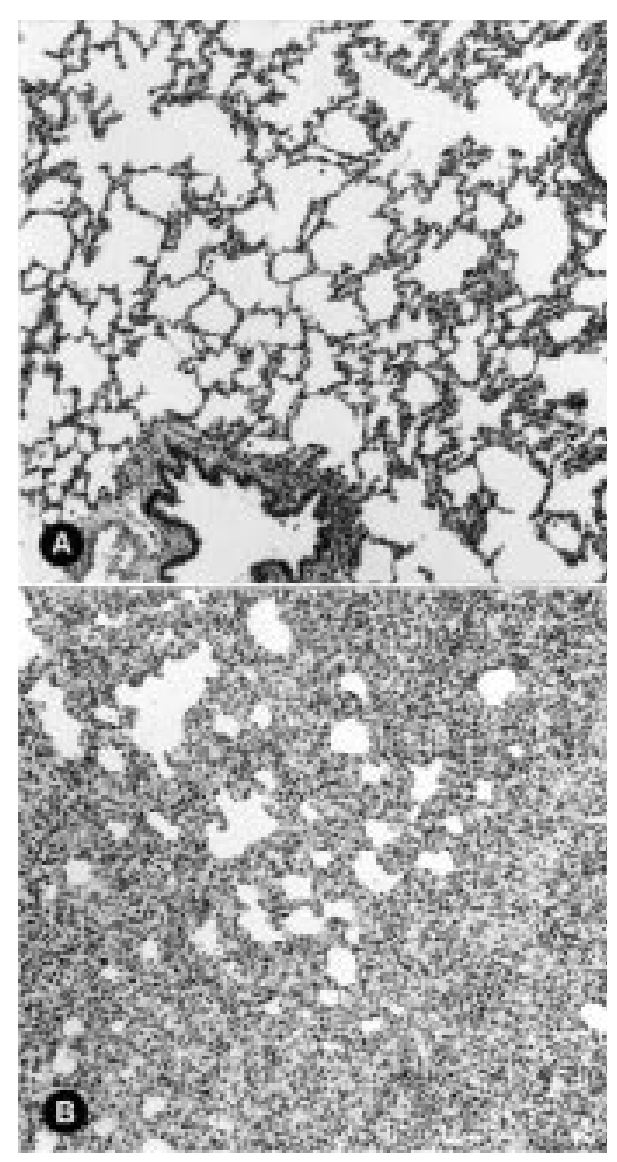

Figure 3. Microscopic appearance of normal lungs (A) of a pig kept as a noninfected control and of extensively consolidated lungs (B) of a pig exposed to a highly virulent (atypical) strain of porcine reproductive and respiratory syndrome virus 2 weeks before necropsy. Both pigs were about 5 weeks old at the time of necropsy.

\subsection{Sample selection}

During the initial experimental studies of PRRS, in which naive gilts were exposed to PRRSV late in gestation, the presence of passively (colostrally) acquired antibody appeared to have little if any inhibitory effect on virus isolation from neonatal pigs. The situation appears to have changed with the common use of attenuated vaccines. Our hypothesis is that although vaccines may sometimes fail to provide complete protection, they prime the immune system so that on subsequent exposure to virulent virus a substantial level of neutralizing antibody (which is typically slow to appear after first exposure to PRRSV) is elicited. The transfer of such antibody via colostrum makes it less likely that the virus will be isolated from the serum of suckling pigs. As a consequence serum samples should, if possible, be obtained from pigs before ingestion of colostrum. If presuckling serum is not available, and pigs are at least several days of age, a more likely sample to yield virus can be obtained by lung lavage. Vaccination also seems to make it more difficult to isolate virulent virus from the sera of clinically affected gilts and sows, even when samples are obtained at or near the time of PRRSV-induced abortion.

The emergence of more virulent strains of PRRSV has introduced another important consideration in regard to sample selection during cases of reproductive failure. Before atypical PRRS, abortions were mostly during late stages of gestation and it was common to isolate PRRSV from at least some of the aborted fetuses and premature pigs. Within the last few years, however, many epidemics of reproductive failure are presented as abortions at all stages of gestation. Although PRRSV is still isolated from at least some of the late-term aborted fetuses and weak, liveborn pigs, and from some of the sera collected from gilts and sows soon after abortion, it is rarely isolated from fetuses aborted earlier in gestation. We assume that these cases of "early" abortion are independent of transplacental infection and are the result of an acute systemic reaction. The practical implication of these findings is that if the number of samples is an issue it is probably prudent to collect at least most samples destined for diagnostic testing from late-term aborted fetuses, stillborn pigs, and weakborn (presuckling) live pigs rather than from fetuses aborted early in 


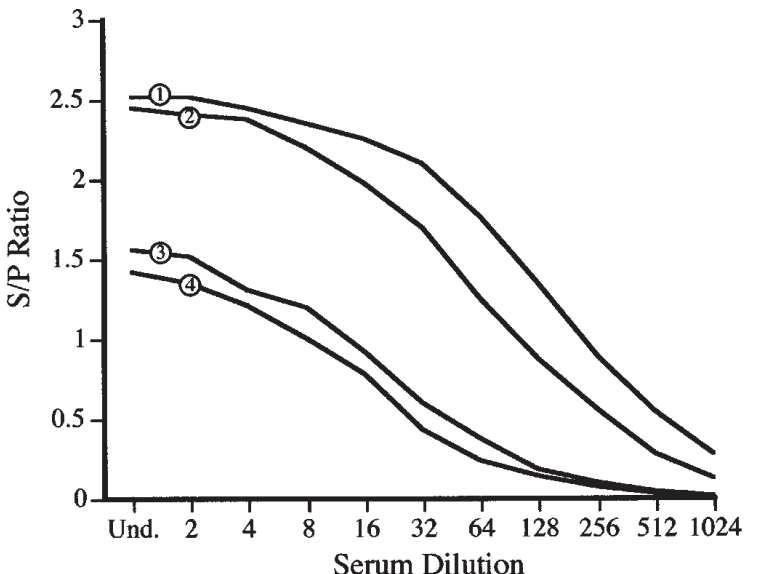

Figure 4. The effect of dilution on the $\mathrm{S} / \mathrm{P}$ ratio determined by enzymelinked immunoassay for porcine reproductive and respiratory syndrome virus (PRRSV). Tests were run on serums collected from gilts 14 days after farrowing. Gilts 1 and 2 were vaccinated twice (at monthly intervals before conception) against PRRSV with a commercial attenuated-live-virus vaccine and challengeexposed to a virulent strain of PRRSV at or about day 90 of gestation. Gilts 3 and 4 were not vaccinated, but were challenged-exposed to the same virulent strain of PRRSV at or about day 90 of gestation. gestation. Regardless of the type of sample, it should be collected and maintained under conditions that minimize virus inactivation.

\subsection{Antibody detection}

Indirect immunofluorescence (IFA), indirect immunoperoxidase monolayer assay (IPMA) and enzyme-linked, immunosorbent assay (ELISA) are the serologic tests used most often to detect and quantitate antibody to PRRSV. ELISA is used less often than either IFA or IPMA for titration because titers ( $\mathrm{S} / \mathrm{P}$ ratios) are not linearly related to relative concentrations of antibody (Fig. 4). On the other hand ELISA is more broadly reactive than either IFA or IPMA and is therefore less likely to miss detecting antibody to a particular strain of the virus. Unfortunately, none of the aforementioned serologic tests can be used as a direct measure of the level of protective immunity. For example, the highest antibody titer detected by any of the three tests is often present within a few weeks after vaccination, but immunity typically takes longer to develop. Compared to IFA, IPMA, and ELISA, the virus neutralization (VN) test is less often used to detect and titrate antibody because it is generally more diffi- cult, expensive, and time consuming to perform, and because VN antibodies are typically slow to appear following vaccination or natural exposure to PRRSV. However, VN antibody may be the best indication of protective immunity, at least to the same strain, or strains, of PRRSV to which the pig was previously exposed.

\subsection{Antigen detection}

Immunohistochemistry and immunofluorescence are useful tests for detecting PRRSV antigens in tissues obtained from infected pigs. They are used diagnostically in some laboratories. However, their application may be somewhat limited because of the level of technical skill required for their satisfactory performance and the experience required for their correct interpretation. They have been especially useful in experimental studies on the pathogenesis of PRRS.

\subsection{Viral nucleic acid detection}

The polymerase chain reaction (PCR) and in situ hybridization (ISH) are tests that have been used successfully to detect the PRRSV genome in infected tissues (ISH) 
and fluids (PCR). PCR is indicated for samples that are toxic for cell cultures and for samples in which PRRSV has been inactivated or is neutralized by antibody. In some laboratories PCR is the test used almost exclusively for confirming suspected cases of PRRS. The ISH technique, however, has found its greatest application in experimental studies designed to identify the cell and tissue tropism of PRRSV during persistent infection.

\subsection{Infectious virus detection}

Virus isolation (VI) is the most commonly used test for detecting PRRSV in diagnostic samples, and, in our opinion, it is, under most circumstances, the test of choice for confirming the presence of the virus. Field experiences as well as several experimental studies have indicated that European strains of PRRSV are most successfully isolated in porcine alveolar macrophages, whereas at least most North American strains can be initially isolated in an established cell line (e.g. MA-104 and MARC-145 cell lines). In most instances the potential problems one might encounter in VI can be circumvented by appropriate sample collection as described in a previous section. The most notable exception is boar semen. Because of the toxicity of boar semen for cell cultures, PCR is usually the only means by which PRRSV can be identified other than by inoculating susceptible pigs with the sample in question. The relative sensitivity of VI and PCR for virus detection depends on the type and condition of the sample, the strain of PRRSV involved, and other issues that are, collectively, too complex for discussion in this brief review.

In our experience one of the most sensitive means to demonstrate the presence of infectious PRRSV in a diagnostic sample has been to administer the sample to a naive, neonatal pig and subsequently collect samples from the pig for attempted virus isola- tion in cell culture. While this procedure is too expensive for routine diagnostic purposes it has been useful in our laboratory to help explain certain findings. For example, during a suspected field epidemic of atypical PRRS, blood samples were collected from 8 gilts that had aborted within the previous 24 hours. The virus was isolated from 2 of the 8 samples by direct inoculation of cell cultures and from 6 of the 8 samples by the first step of pig inoculation. Assuming the abortions were caused by PRRSV, the latter, that is detection of PRRSV in 6 of the 8 samples, seemed more consistent with our expectations. Although we are not sure how to explain these discrepancies, the fact that the gilts had been vaccinated before exposure to field virus suggests that circulating antibody may have had a role. Whatever the reason, the importance of collecting a number of samples for testing is emphasized.

\section{STRAIN IDENTIFICATION}

A presumptive identification of the strain of PRRSV detected in a particular sample has become an important component of PRRS diagnosis in North America. It provides additional information in epidemiologic investigations and in determining the possible persistence and clinical relevance of vaccine virus in vaccinated herds.

Differential tests. The most commonly used tests for strain identity are restriction fragment length polymorphism (RFLP) analysis [2, 5] and base sequencing [3]. The RFLP analysis, as performed in our laboratory, involves conversion (reverse transcription) of open reading frame (ORF) 5 and short contiguous sequences of viral RNA to double-stranded DNA followed by nonnested-set PCR amplification and restriction endonuclease digestion of the amplified DNA. Occasionally, for specific purposes, other ORFs are converted, amplified, and digested [2]. In a recent modification of the procedure its timeliness, and in some 
cases its sensitivity, were improved using a nested-set PCR to amplify the relevant portion of the viral genome [4]. Base sequencing often involves additional segments of the viral genome (e.g. ORF6 in addition to ORF5). Panels of monoclonal antibodies have also been used to provide presumptive strain identification [6]. Despite the usefulness of these tests it must be kept in mind that all focus on only a small portion of the viral genome and therefore none, by themselves, provide definitive evidence of strain characteristics, such as virulence, or ancestry.

Disclaimer: No endorsements are herein implied. Brand names are necessary to report factually on available data; however, the USDA neither guarantees nor warrants the standards of the products, and the use of the names by the USDA implies no approval of the products to the exclusion of others that may also be suitable.

\section{REFERENCES}

[1] Benfield D.A., Collins J.E., Dee S.A., Halbur P.G., Joo H.S., Lager K.M., Mengeling W.L., Murtaugh M.P., Rossow K.D., Stevenson G.W., Zimmerman J.J., Porcine reproductive and res- piratory syndrome, in: Straw B.E., D'Allaire S., Mengeling W.L., Taylor D.J. (Eds.), Diseases of Swine, Iowa State University Press, Ames, Iowa, 1999, pp. 201-232.

[2] Mengeling W.L., Vorwald A.C., Lager K.M., Clouser D.E., Wesley R.D., Identification and clinical assessment of suspected vaccine-related field strains of porcine reproductive and respiratory syndrome virus, Am. J. Vet. Res. 60 (1999) 334-340.

[3] Murtaugh M.P., Faaberg K.S., Yuan S., Kapur V., Interrelatedness of PRRS virus isolates in North America, Proc. 24th Allen D. Leman Conf. (1997) 146-149.

[4] Umthun A.R., Mengeling W.L., Restriction fragment length polymorphism analysis of strains of porcine reproductive and respiratory syndrome virus by use of a nested-set reverse transcriptase-polymerase chain reaction, Am. J. Vet. Res. 60 (1999) 802-806.

[5] Wesley R.D., Mengeling W.L., Andreyev V., Lager, K.M., Differentiation of vaccine (Strain RespPRRS) and field strains of porcine reproductive and respiratory syndrome virus by restriction enzyme analysis, Proc. 27th Annu. Meet. Am. Assoc. Swine Practitioners, March 2-5, Nashville, Tennessee, USA (1996) 141-143.

[6] Yang L., Yoon K.J., Zimmerman J.J., Frey M.L., Harmon K.M., Platt K.B., Antigenic and genetic variations of the $15 \mathrm{kD}$ nucleocapsid protein of porcine reproductive and respiratory syndrome virus isolates, Arch. Virol. 144 (1999) 525-546.

[7] Zimmerman J.J., Epperson W., Wills R.W., McKean J.D., Results of the recent survey of the membership of the AASP for outbreaks of sow abortion and mortality, Swine Health and Production 5 (1997) 74-75. 